\title{
L'épiscopat québécois au moment de la formation du diocèse de Sherbrooke (1874)
}

\section{Nive Voisine}

Volume 41, 1974

URI : https://id.erudit.org/iderudit/1007240ar

DOI : https://doi.org/10.7202/1007240ar

Aller au sommaire du numéro

Éditeur(s)

Les Éditions Historia Ecclesiæ Catholicæ Canadensis Inc.

ISSN

0318-6172 (imprimé)

1927-7067 (numérique)

Découvrir la revue

Citer cet article

Voisine, N. (1974). L'épiscopat québécois au moment de la formation du diocèse de Sherbrooke (1874). Sessions d'étude - Société canadienne d'histoire de l'Église catholique, 41, 25-41. https://doi.org/10.7202/1007240ar

Tous droits réservés @ Les Éditions Historia Ecclesiæ Catholicæ Canadensis Inc., 1975
Ce document est protégé par la loi sur le droit d'auteur. L'utilisation des services d'Érudit (y compris la reproduction) est assujettie à sa politique d'utilisation que vous pouvez consulter en ligne.

https://apropos.erudit.org/fr/usagers/politique-dutilisation/ 


\section{L'épiscopat québécois au moment de la formation du diocèse de Sherbrooke (1874)}

Fêter le $200^{\mathrm{e}}$ anniversaire du diocèse de Québec par la fondation de celui de Sherbrooke n'avait pas été une mesure prévue d'avance. On pensait établir un siège épiscopal dans les Cantons de l'Est depuis longtemps. Déjà, en 1868, la rumeur s'accréditait que les Pères du Concile de Québec se préoccupaient de ce problème ${ }^{1}$ et, au début de 1869, l'Écho du cabinet de lecture paroissial de Montréal relançait la nouvelle en soulignant qu'

Un siège épiscopal de ce côté ne manquera pas de donner un nouvel élan à la religion et même ne servira pas peu à encourager les colons à persévérer dans leurs travaux de défrichement et à donner naissance à de nouvelles paroisses qui y attireront encore de nouveaux pionniers : c'est donc une œuvre civilisatrice en même temps que religieuse ${ }^{2}$.

L'assemblée épiscopale discutait du problème en $1872^{3}$ et communiquait sa décision à Rome qui l'entérinait en érigeant le diocèse de Sherbrooke le 28 août 1874.

Quant aux fêtes du deuxième anniversaire, elles survenaient en un moment difficile. Depuis de nombreuses années, l'épiscopat de la province ecclésiastique de Québec était divisé, mais ce club sélect et respectable n'en avait laissé rien filtrer pendant longtemps. Les prélats cependant se plaignaient entre eux de la situation. Un observateur lointain, comme $\mathrm{M}^{\mathrm{gr}}$ Alexandre Taché, de Saint-Boniface, ne pouvait s'empêcher de soupirer : "Nous aurions besoin d'être plus unis et l'on se divise d'avantage [sic] chaque jour ${ }^{4}$ » et, en 1868 , toujours à son ami Laflèche, il confiait :

Va sans dire qu'il m'eut été bien doux de voir tous les Pères du Concile de Québec cependant je vous avouerai naïvement

\footnotetext{
3 Mgr E.-A. Taschereau à Mgr Ls-Fr. Laflèche, 2 mai 1872, Archives
} p. 63.

1 «Concile de Québec», Le Journal de Québec, 9 mai 1868, p. 2.

2 L'Écho du cabinet de lecture paroissial de Montréal, XI (1869), du Séminaire de Trois-Rivières (ASTR), Fonds Laflèche, A 1 T 99-39.

4 Mgr A. Taché à Mgr Laflèche, 30 sept. 1867, ibid., D 1 T 144-19. 
que la désunion qui semble exister entre les Évêques du Canada est pour moi une source de peine bien vive et que je redoutais de me trouver à leur assemblée 5 .

En ce moment, l'évêque de Montréal, $\mathrm{M}^{\mathrm{gr}}$ Ignace Bourget, était au centre des querelles, à cause de ses difficultés avec les sulpiciens et de ses demandes réitérées d'une université catholique à Montréal. Graduellement le climat s'était détérioré avec la bataille autour du Programme catholique de 1871, le projet de loi pour permettre aux jésuites de fonder une université, les incidents des noces d'or de $\mathrm{M}^{\mathrm{gr}}$ Bourget, le voyage des seigneurs Taschereau et Laflèche à Rome en 1872, etc. J'exagère à peine en disant que l'assemblée des évêques était devenue une foire d'empoigne et que régulièrement deux clans s'affrontaient : $\mathbf{M}^{\mathrm{gr}}$ Taschereau, de Québec, $\mathbf{M}^{\mathrm{gr}}$ Charles La Rocque, de Saint-Hyacinthe et $\mathbf{M}^{\mathrm{gr}}$ Jean Langevin, de Rimouski, d'une part; et, d'autre part, $\mathbf{M}^{\mathrm{gr}}$ Bourget, de Montréal et $\mathbf{M}^{\mathrm{gr}}$ Laflèche, de TroisRivières. (Je laisse de côté $\mathbf{M}^{\mathrm{gr}} \mathrm{J}$ Joseph-Bruno Guigues, d'Ottawa, qui essaie d'ordinaire de se tenir en dehors des conflits.)

Dans ces conditions difficiles, $\mathbf{M}^{\mathbf{g r}}$ Taschereau avait quelque mérite à vouloir célébrer le $200^{\mathrm{e}}$ anniversaire de l'érection du siège de Québec par une réunion d'évêques; il ne fallait pas que les incidents de 1872 à Montréal se répètent. Peut-être sans le savoir, il fut malin en invitant "les cinquante-neuf évêques, dont les diocèses ont autrefois fait partie de celui de Québec, à venir rendre grâces avec nous et à unir leurs prières aux nôtres dans cette circonstance solennelle ${ }^{6} \%$. La fête eut lieu le dimanche $1^{\text {er }}$ octobre. Si l'on en croit les journaux de Québec, ce fut « la plus imposante de toutes les manifestations religieuses » jamais vues à Québec.

Jamais illumination n'aura été aussi brillante et aussi générale; jamais nous n'aurons vu tant de chefs d'Églises et tant de lévites réunis dans nos murs; jamais les décorations de tous les genres n'auront été aussi belles et aussi nombreuses ?.

Quant aux évêques, ils étaient moins nombreux que prévus : une vingtaine seulement; $\mathbf{M}^{\mathrm{gr}}$ Bourget était le seul absent de la province ecclésiastique de Québec.

5 Le même au même, 17 sept. 1868, ibid., D 1 T 144-22.

6 «Mandement à l'occasion du deux centième anniversaire de l'érection du Siège de Québec 》, 8 sept. 1874, Mandements, lettres pastorales et circulaires des évêques de Québec, Son éminence le cardinal Taschereau, I, p. 220.

7 Le Journal de Québec, 29 sept. 1874, p. 2; 2 oct. 1874, p. 2. 
Ces prélats qui oublient un moment leurs dissensions pour fêter deux cents ans d'histoire, qui sont-ils ? Que se cache-t-il derrière les noms de Taschereau, de Bourget, de Laflèche, de Langevin, et de quelques autres ? C'est ce dont j'aimerais vous entretenir avant d'essayer de caractériser dans son ensemble l'épiscopat québécois de 1874.

\section{I. -L'épiscopat québécois : les personnalités}

En 1874, la province ecclésiastique de Québec comprend, outre Québec, les diocèses de Montréal, de Saint-Hyacinthe, de TroisRivières, de Rimouski et aussi d'Ottawa. Les titulaires en sont : pour Québec, $\mathbf{M}^{\mathrm{gr}}$ Elzéar-Alexandre Taschereau (1871); pour Montréal, M ${ }^{\mathrm{gr}}$ Ignace Bourget (1840), avec $\mathrm{M}^{\mathrm{gr}}$ Édouard-Charles Fabre (1873) comme coadjuteur; pour Saint-Hyacinthe, $M^{\mathrm{gr}}$ Charles La Rocque (1866); pour Rimouski, Mrr Jean Langevin (1867) et, pour Ottawa, M ${ }^{\mathrm{gr}} \mathrm{J}$.-Bruno Guigues (1847).

\section{1) Mgr Elzéar-Alexandre Taschereau (1820-1898)}

L'archevêque Taschereau est peut-être, aujourd'hui, le moins connu de ce groupe important. Nous attendons et souhaitons l'étude sérieuse qui nous révélera les aspects nombreux de cette personnalité controversée.

Né en 1820, l'archevêque a donc 54 ans en 1874, mais il n'est en fonction que depuis 1871 , le dernier à avoir pris son poste dans ce corps où l'âge et le « rang de doyen 》 ont une certaine importance. Faut-il rappeler, cependant, que ses relations avec l'épiscopat datent de beaucoup plus tôt ?

C'est un homme transcendant. Quand $\mathbf{M}^{\mathrm{gr}}$ Charles-François Baillargeon l'a présenté à ses collègues et à Rome comme le plus digne de lui succéder, il a insisté sur sa science théologique (droit canonique, théologie, écriture sainte) et sur ses connaissances en littérature, en philosophie, etc. ${ }^{8}$. Il avait raison, car par ses études, son enseignement, la fréquentation du milieu universitaire, Taschereau avait acquis une culture théologique et profane qui tranchait parfois sur celle de ses collègues et qui n'était pas sans influencer sa vision des problèmes du moment. Les évêques, les premiers, le reconnaissaient depuis longtemps et, en $1867, \mathrm{M}^{\mathrm{gr}}$ Laflèche écrivait à $\mathrm{M}^{\mathrm{gr}}$

8 Mgr C.-F. Baillargeon, [Liste des candidats à sa succession], 27 oct. 1867, ASTR, Fonds Laflèche, A 1 B 10-16. 
Baillargeon : «Vous avez auprès de vous un savant dont je respecte beaucoup les opinions ${ }^{9} 》$. Faut-il ajouter que Taschereau parlait couramment l'italien, ce qui pouvait lui ouvrir la porte de quelques monsignori à Rome?

Cet intellectuel est, aux yeux de $\mathrm{M}^{\mathrm{gr}}$ Baillargeon, sans défaut :

Mr. Taschereau est certainement doué de talents transcendants, d'une haute intelligence, d'une grande pénétration d'esprit, d'un jugement sain, d'un grand amour pour le travail, d'une remarquable expédition dans tout ce qu'il fait, et d'un rare esprit d'ordre en toutes choses. D'ailleurs, plein de courage, ferme et énergique, il possède à mon avis toutes les qualités nécessaires pour bien administrer les choses spirituelles et temporelles d'un diocèse comme celui de Québec.

A toutes ces belles qualités, naturelles et acquises, Mr. Taschereau joint une foi vive, une haute piété, une scrupuleuse exactitude dans l'accomplissement de tous ces devoirs, dans l'observation des moindres règles de la discipline ecclésiastique, un zèle sincère pour la gloire de Dieu, l'honneur de l'église et le salut des âmes, enfin une grande pureté de conscience, et le mérite d'une vie sans tache et sans reproche depuis son enfance 10.

Après un tel panégyrique, $\mathbf{M}^{\mathrm{gr}}$ Taschereau a plus de chances d'être canonisé que $\mathrm{M}^{\mathrm{gr}}$ de Laval! Il faut comprendre que, pour faire passer son candidat, $\mathbf{M}^{\mathrm{gr}}$ Baillargeon ne pouvait pas s'embarrasser de nuances. Le portrait devrait sûrement être retouché, mais il serait trop long de le faire aujourd'hui. Je me contenterai donc de quelques précisions.

Les adversaires de Taschereau lui reprochent particulièrement deux choses : son manque de zèle à combattre l'erreur, sa « froideur » dans le commerce de la vie. Je laisse évidemment de côté les accusations de libéralisme portées contre lui; elles tombent d'elles-mêmes quand on lit ses écrits. Mais les ultramontains lui reprochent de pactiser avec les libéraux (membres du parti libéral, comme Joseph Cauchon, ou professeurs de Laval) et de ne rien faire pour les ramener à l'orthodoxie ou condamner leurs journaux. Un groupe de prêtres du diocèse de Québec écrivent dans un mémoire de 1873 :

$9 M^{g r}$ Laflèche à Mgr C.-Fr. Baillargeon, 27 nov. 1867, Archives de l'évêché de Trois-Rivières (AETR), Registre des lettres, V, 9 B.

$10 \mathrm{Mgr}$ Baillargeon, [Liste des candidats à sa succession], 27 oct. 1867, ASTR, Fonds Laflèche, A 1 B 10-16. 
Il avait d'abord prouvé en plusieurs cas qu'il n'avait point la rectitude dans le jugement, car tout en professant, au moins extérieurement, les vrais principes sur des questions importantes, il en avait fait ou laissé faire, pouvant l'empêcher, des applications absolument fausses... ${ }^{11}$.

Dans un mémoire de $1882, \mathrm{M}^{\mathrm{gr}}$ Laflèche soutient que * la véritable cause [de nos difficultés] se trouve dans les influences diverses que subit à son insu, je pense bien, $M^{\text {gr }}$ l'Archevêque, et qui l'inclinent tantôt à marcher avec ses suffragants, et tantôt à favoriser les libéraux ${ }^{12}$ ». À propos du Programme catholique, Laflèche note dans le même document :

Et le Métropolitain si sévère à l'égard du Journal des TroisRivières et du Nouveau-Monde qui ne relevaient point de sa juridiction, n'eût pas un mot de blâme pour défendre ses collègues contre de telles injures qui s'imprimaient à la porte de son palais dans un journal entièrement sous sa surveillance ${ }^{13}$.

On pourrait multiplier les témoignages semblables, mais ce serait inutile. Il vaut mieux, je crois, bien souligner ceci : Bourget et Laflèche, et parfois les autres suffragants, n'acceptent pas le style de direction pastorale de Taschereau. Eux que la passion anime, qui se veulent des soldats, des chevaliers, des croisés combattant les idées modernes, ils ne comprennent pas leur archevêque qui n'aime guère parler, prêcher ou écrire; son silence leur apparaît facilement un manque de zèle. Ils n'acceptent pas plus sa recherche des ménagements, car, à leurs yeux, elle conduit à pactiser avec le Mal !

Je n'insiste pas sur le caractère marmoréen de la personnalité de Taschereau; tout le monde en parle, sans l'interpréter de la même façon. Le groupe de prêtres, dont j'ai parlé plus haut, rappellent que l'archevêque est * très-froid et très-raide dans le commerce ordinaire de la vie », mais c'est pour ajouter qu' « il manquait cependant

11 [Mémoire d'un groupe de prêtres du diocèse de Québec], 6 janv. 1873, ibid.. A 1 T 100-1.

12 Mémoire de l'évêque des Trois-Rivières sur les difficultés religieuses en Canada, Rome, Imprimerie de Rome, 1882, p. 43. Quelques lignes plus bas, Laflèche rapporte ce jugement de Wilfrid Laurier : «Monseigneur l'Archevêque nous rend certainement service en inclinant tantôt d'un côté et tantôt de l'autre; mais il n'est pas l'homme qu'il nous faut pour un triomphe complet, il est trop girouette ».

13 Ibid., p. 38s. 
de véritable énergie dans les circonstances où il fallait en déployer ${ }^{14}$ ». Hector Langevin, qui a sollicité une entrevue, reçoit comme réponse cette seule phrase: "Monsieur, je serai à ma chambre toute cette après-midi ». Le ministre conservateur explose : " Notre cher Archevêque aura besoin de se polisser un peu, s'il n'a pas envie qu'on n'ait aucuns rapports avec lui. C'est une vraie porte de prison ${ }^{15}$ 》. Que de témoignages semblables sur son manque de conversation, son laconisme, sa froideur, son arrogance, etc. Alexis Pelletier y met le bouquet avec ironie et méchanceté; dans ses Croquis de topographie universitaire, il décrit allègrement l'archevêque et la ville épiscopale :

Là, cependant, se rencontre le type de la domination, de la supériorité arrogante, de l'autocratie impérieuse et arbitraire, d'un froid dédain mal déguisé pour toute autre grandeur que la sienne, d'une crainte malsaine de descendre de cette hauteur... la fière Métropole entend bien que personne ne soit assez osé pour douter d'elle, qu'on ne cesse de l'admirer avec tous ses beaux atours anciens et nouveaux; qu'on s'incline humblement devant elle; même devant ses parvenus incapables de soutenir son antique gloire; qu'on s'honore d'être admonesté par elle, instruit par elle, gouverné par elle, jugé par elle... ${ }^{16}$.

Ces éléments sont sans doute importants pour comprendre l'action de Taschereau et ses difficultés avec les autres membres de l'épiscopat. Mais deux faits éclairent encore davantage son comportement.

Le premier est, si l'on peut dire, son intégration au séminaire et à l'université. De l'âge de huit ans jusqu'à sa nomination au poste d'archevêque, il a été élève, professeur, directeur, supérieur, recteur de cette institution et sa vie s'est confondue avec les heurs et malheurs de cette maison. Ayant participé à la fondation de l'université, il en a fait pour ainsi dire une ouvre personnelle et l'a défendue avec âpreté comme recteur et comme archevêque. Dès qu'on l'attaque, il s'élève pour riposter, et il fait des pieds et des mains pour assurer à la fois sa survie et son monopole (pour lui,

14 [Mémoire d'un groupe de prêtres du diocèse de Québec], 6 janv. 1873, ASTR, Fonds Laflèche, A 1 T 100-1.

$15 \mathrm{H}$. Langevin à Mgr Jean Langevin, 17 oct. 1872, Archives nationales du Québec (ANQ), Fonds Langevin, B 5,765.

16 Cité dans Thomas Charland, «Un gaumiste canadien : l'abbé Alexis Pelletier », Revue d'histoire de l'Amérique française, I, 2 (sept. 1947), p. 223. 
l'une ne va pas sans l'autre). De là, entre autres choses, ses attaques virulentes contre $\mathbf{M}^{\mathrm{gr}}$ Bourget et son projet d'une université catholique à Montréal, le travail de harassement contre $\mathrm{M}^{\mathrm{gr}}$ Laflèche qui prend trop ouvertement parti pour Montréal, la protection qu'il assure aux professeurs «libéraux 》 de Laval et aux journaux qui les défendent (ce qui l'entraîne, en 1876, à affronter tous ses suffragants...), le « lobbying 》 permanent à Rome où les moyens employés n'ont pas toujours la pureté du cristal. Je crois que Taschereau était prêt à tout pour protéger le séminaire et l'université, même à écrire une longue lettre pastorale contre le libéralisme en 1875 !

Le deuxième fait est la nécessité de s'affirmer comme archevêque. Quand il monte sur le trône de Québec, le leadership appartient alors à Montréal par suite de diverses circonstances comme la longue maladie de $\mathrm{M}^{\mathrm{gr}}$ Pierre-Flavien Turgeon et le caractère de $M^{\mathrm{gr}}$ Baillargeon. Sans oublier, bien sûr, la personnalité envahissante de $\mathrm{M}^{\mathrm{gr}}$ Bourget. Pour rétablir la situation, Taschereau doit donc s'affirmer le plus tôt possible et lutter inlassablement contre l'érosion de son pouvoir d'archevêque. C'est là, je crois, l'explication de son intervention (trop) rapide dans la question du Programme catholique: se taire et laisser $\mathrm{M}^{\mathrm{gr}}$ Bourget et $\mathrm{M}^{\mathrm{gr}}$ Laflèche profiter de cette nouvelle croisade ultramontaine, c'est appuyer leurs tactiques et se mettre à leur remorque. Et Taschereau, par dessus le marché, n'est pas du tout d'accord avec le contenu du programme. Voilà pourquoi, avec la fougue d'un nouvel ordonné, bien peu de temps après son sacre, il condamne le Programme catholique, censurant indirectement Bourget et Laflèche et révélant à tout le monde la division qui existe au sein de l'épiscopat. Sans doute le geste est-il imprudent, - en 1874, le cardinal Patrizzi lui écrit que les cardinaux " n'ont pas cru devoir approuver votre conduite peut-être trop précipitée ${ }^{17}$ 》, - mais c'est l'occasion rêvée pour montrer qu'il y a désormais un vrai archevêque à Québec.

Enfin, au point de vue des idées, il faut souligner que, tout en ne s'éloignant guère de ses collègues, Taschereau veut éviter l'intransigeance. Vivant dans une société plus ouverte, - à cause du milieu politique de la capitale et de l'influence universitaire, - il croit sans doute aux déclarations doctrinales, mais il est plus sensible aux

17 Card. Patrizzi à Mgr Taschereau, 4 août 1874, Mémoire de l'évêque des Trois-Rivières sur les difficultés religieuses en Canada, Pièces justificatives, p. 46. 
situations concrètes, aux questions qu'il appelle lui-même "pratiquement pratiques $\gg{ }^{18}$. Il s'en est admirablement bien expliqué dans une lettre à Siméon Pagnuelo à propos des Etudes historiques et légales sur la liberté religieuse en Canada:

On est exposé, lui écrit-il, à prendre pour vérité absolue ce qui est matière d'opinion; on se laisse parfois entraîner à mal noter ce que l'Église n'a pas encore jugé à propos de condamner; l'idéal de ce qui devrait être tend à faire oublier la réalité; un avenir que l'on souhaite avec impatience empêche de compter avec un passé et un présent hérissé de difficulté.

Il ajoutera, en 1881, dans une lettre à $\mathrm{M}^{\mathrm{gr}}$ Laflèche : «Il faut prendre les hommes non pas tels qu'ils devraient être, mais tels qu'ils sont ${ }^{19}$ ». Admettez qu'il n'y a pas beaucoup d'évêques canadiens qui ont tenu de tels propos au XIX ${ }^{e}$ siècle.

\section{2) Mgr Ignace Bourget (1799-1885)}

En face de $\mathbf{M}^{\mathrm{gr}}$ Taschereau, nous trouvons régulièrement $\mathbf{M}^{\mathrm{gr}}$ Bourget et $\mathbf{M}^{\mathrm{gr}}$ Laflèche.

Âgé de 75 ans et évêque de Montréal depuis 1840, $\mathrm{M}^{\mathrm{gr}}$ Bourget est de loin le doyen de l'épiscopat. C'est un géant, l'une des plus grandes figures du $\mathrm{XIX}^{\mathrm{e}}$ siècle canadien. Il a été un créateur. Après avoir réveillé les forces catholiques par un mouvement de réforme qui débute avec son règne, il multiplie les auvres dans tous les domaines : enseignement, bien-être, colonisation, liturgie, loisirs ('CEuvre des bons livres), etc. Il est partout et son prosélytisme rayonne en dehors de son diocèse. Ses initiatives dérangent bien du monde et il n'y a pas que ses ennemis qui trouvent qu'il s'occupe de trop d'affaires ou qu'il va trop vite en besogne. Il est symptomatique que l'abbé Taschereau écrive au grand vicaire Laflèche en 1865 : "Voilà donc Mgr de Montréal revenu chez lui. Espérons qu'il va se tenir en paix et qu'il y laissera les autres ${ }^{20}$. Bourget a été aussi un lutteur. $\mathrm{M}^{\mathrm{gr}}$ Lafièche en donne le témoignage à Rome en 1876 :

Depuis près de 40 ans Mgr. Bourget est sur la brèche pour revendiquer et défendre les droits de l'Église, il a consacré

18 Mgr Taschereau à l'abbé Charles-Olivier Caron, 14 oct. 1876, ASTR, Fonds Laflèche, A 1 T 100-65.

19 Mgr Taschereau à Mgr Laflèche, 21 avril 1881, ibid., A 1 T 101-34.

20 Le même au même, 20 déc. 1865, ibid., A 1 T 99-4. 
tout ce qu'il avait d'activité et d'énergie à la défense de la vérité, à combattre et repousser l'erreur. Et ce n'est pas par choix qu'il s'est ainsi trouvé en évidence sur la brèche, et, pour ainsi dire, comme à la tête des défenseurs de la religion et de la vérité, c'est la position même que lui a fait la Divine Providence en le plaçant à la tête du vaste et populeux diocèse de Montréal, et qui lui a imposé cette rude tâche ${ }^{21}$.

Il s'est attaqué avec acharnement à de gros morceaux : aux libéraux de l'Institut canadien; aux sulpiciens qui avaient déjà mené la vie dure à $\mathrm{M}^{\mathrm{gr}}$ Jean-Jacques Lartigue, son prédécesseur; aux Messieurs du Séminaire et de l'université Laval de Québec qui, au dire d'Alexis Pelletier, \&veulent être les seules lumières qui éclairent tout canadien venant en ce monde 22 » Dans ces combats, il a épuisé ses forces et sa santé, et il s'est créé de solides inimitiés.

L'une des plus fortes est celle des professeurs de Québec, et nommément celle de Taschereau. Avec les abbés Benjamin Pâquet, E.-G. Plante et Dominique Racine, il est sans doute le plus grand contempteur de l'évêque de Montréal. Dans la longue querelle à propos de l'université, il s'emploie avec succès à miner la crédibilité de son adversaire auprès des autorités romaines, surtout auprès du cardinal Barnabo, préfet de la Propagande. C'est avec étonnement qu'on lit les jugements de Québec sur $\mathbf{M}^{\mathrm{gr}}$ Bourget. On le reconnaît pour un saint, mais un saint niais ${ }^{23}$, un saint fou. Benjamin Pâquet écrit en 1864 : «En 1862 Mgr. de Montréal est parti de Rome avec la réputation de saint, mais cette fois-ci tout présage qu'il partira avec la réputation d'un saint fou ${ }^{24}$ ». L'abbé Dominique Racine, tout au contraire, le compare à un brigand :

À Québec, écrit-il à l'abbé T.-E. Hamel, on a toujours peur de faire du mal aux autres, même quand on y défend sa peau; aussi vous vous faites râcler parfois d'une rude manière. Quand on est assailli par un brigand, on se défend et on ne pêche [sic] pas 25 .

21 Laflèche au card. Franchi, 11 août 1876, AETR, Registre des lettres, VI b, p. 160.

22 A. Pelletier, La Source du mal de l'époque, dans A. Savaète, Voix canadiennes, Vers l'abime, t. III, Paris, Arthur Savaète, (s.d.), p. 89.

23 Benjamin Paquet à E.-G. Plante, 27 déc. 1864, Archives du Séminaire de Québec (ASQ), cart. Plante, 36.

24 Ibid.

25 Dominique Racine à T.-E. Hamel, 7 avril 1863, ibid., Un. 80-31. 
François Langelier insiste davantage sur son ignorance : «Ce cher évêque de Montréal entend l'organisation d'une université comme j'entends le Chinois ». Au dire des gens de Québec, le cardinal Barnabo serait dans les mêmes sentiments. Selon Taschereau,

il paraît avoir une dent très longue et très pointue contre Mgr. de Montréal. [Il aurait déclaré :] La sainte Église Romaine est notre mère, c'est vrai; mais il ne faut pas troubler l'harmonie pour l'imiter en toutes choses. Parce que ma mère porte une coiffure de femme, est-ce [que] je dois en porter une aussi ? 26

Décidément, on n'aime pas Bourget dans ces milieux! Il ne faut donc pas s'étonner qu'il y ait, entre l'archevêque et son suffragant, une incompatibilité totale que ne voile même pas une froide politesse.

Il faut ajouter, cependant, que $\mathrm{M}^{\mathrm{gr}}$ Bourget n'attire pas que des inimitiés et qu'un très grand nombre de disciples l'ont appuyé et suivi d'une façon inconditionnelle, avec une ferveur sans cesse renouvelée. Mais, en 1874, l'évêque de Montréal est un être vieilli, malade, qui, malgré tout le dynamisme qui l'habite encore, doit penser à la relève. Pour la gouverne du diocèse, ce sera $\mathrm{M}^{\mathrm{gr}}$ ÉdouardCharles Fabre; pour la direction des combats, c'est l'évêque de TroisRivières, $\mathbf{M}^{\mathrm{gr}}$ Louis-François Laflèche.

\section{3) Mgr Louis-François Laflèche (1818-1898)}

Laflèche s'est rangé définitivement dans le clan de Montréal en 1871-72. Auparavant, il est en relations suivies et chaleureuses avec $\mathrm{M}^{\mathrm{gr}}$ Baillargeon, de Québec, et il s'efforce d'atténuer la querelle entre le Journal des Trois-Rivières et l'université Laval. Même la discussion autour du Programme catholique n'empêche pas Taschereau et Lafièche d'échanger des confidences. À Taschereau qui se plaint : «Hélas ! en jetant les yeux sur l'année qui finit, je ne vois que le vide ${ }^{27}$ 》, Laffèche répond avec la même franchise : «[...] de quel sentiment ne me sens-je pas affecté, quand je regarde les 5 années écoulées de mon épiscopat? Je vous avoue que j'en suis par temps effrayé et qu'il me vient en pensée d'envoyer ma résignation au St-Père ${ }^{28}$.

26 Taschereau à Ls-J. Casault, 15 mai 1862, ibid., Un. 102, y.

27 Mgr Taschereau à Mgr Laflèche, 19 mars 1872, ASTR, Fonds Laflèche, A 1 T 99-36.

28 Mgr Laflèche à Mgr Taschereau, 22 mars 1872, AETR, Registre des lettres, $\mathrm{V}, \mathrm{n}^{\circ} 21$. 
Sans doute que le suffragant ne voit pas encore que les principes sont en jeu...

Laflèche franchit le Rubicon à l'automne 1872 quand, en réponse à l'archevêque qui lui annonce son départ pour Rome, il décide d'aller y défendre contradictoirement la position de l'évêque de Montréal. Il s'en explique ainsi à son clergé :

C'est à la demande instante et réitérée du vénérable Évêque de Montréal, d'un grand nombre de prêtres et de fidèles éminents de diverses parties de la Province, que j'en suis arrivé à la conclusion que le bien de la religion et le rétablissement durable de la concorde exigeaient de moi ce sacrifice, malgré le triste état de ma santé 29.

Il est encore plus explicite avec son grand ami $\mathrm{M}^{\mathrm{gr}}$ Alexandre Taché, de Saint-Boniface :

Toutes les questions, lui écrit-il le 13 décembre 1872, peuvent se résumer sous quatre chefs : $1^{\circ}$ Une Université Catholique à Montréal; $2^{\circ}$ L'existence d'une presse Catholique conformément au vœu exprimé par les Pères du Second Concile de Québec dans leur lettre pastorale du 4 juin $1854 ; 3^{\circ}$ Les paroisses canoniques de Montréal, et enfin $4^{\circ}$ Le choix d'un coadjuteur pour Montréal 30.

Ma manière de voir sur toutes ces questions est la même que celle de Monseigneur. [...] sans doute il y a du tort des deux côtés, surtout dans la presse où l'on s'est laissé aller à trop de vivacités, et même à des excès bien regrettables; mais sur le fond des questions, on a tort à Québec, dans mon humble opinion et l'on est dans le vrai à Montréal ${ }^{31}$.

Voilà donc pourquoi, à partir de 1872, Laflèche porte la livrée de Montréal et confond de plus en plus sa cause avec celle de $\mathrm{M}^{\mathrm{gr}}$ Bourget.

Il le fait également parce qu'il est en conformité de pensée avec l'évêque de Montréal. Comme lui, il croit que «la lutte qui

29 «Circulaire au clergé 》, 9 déc. 1872, Mandements, lettres pastorales, circulaires de Mor L. F. Laflèche, second évêque des Trois-Rivières, t. I (18671874), p. 395s. ment dits.

30 Remarquez qu'il n'est pas question des problèmes politiques propre-

31 Mgr Laflèche à Mgr A. Taché, 13 déc. 1872, AETR, Registre des lettres, VI, 14 B. 
partage en deux camps ennemis nos compatriotes, n'est que la grande lutte entre le bien et le mal ${ }^{32}$ », que « le souffle de la grande erreur contemporaine» s'est fait sentir au Québec et que «la voix enchanteresse des sirènes libérales $\gg y$ a fait des victimes ${ }^{33}$, qu'enfin «par ses tendances anti-sociales, par sa haine de l'Église, par ses principes pervers, (le libéralisme canadien) ne diffère en rien du libéralisme européen ${ }^{34}{ }^{\star}$. Ce n'est pas l'avis de Taschereau, mais il affrontera Laflèche à ce sujet après 1874 seulement.

Enfin, faut-il rappeler que, déjà en 1872 , certains pensent que Laflèche se laisse manipuler facilement ? $\mathbf{M}^{\mathrm{gr}}$ La Rocque, de SaintHyacinthe, écrit à $\mathbf{M}^{\mathrm{gr}}$ Jean Langevin : «Sera-t-il vrai que notre digne Frère des Trois-Rivières entreprenne aussi le voyage? Mais le scandale était pourtant déjà assez grand. L'excellent Évêque finira, j'espère, par s'apercevoir que le rôle que l'on veut lui faire accepter, n'est pas assez agréable pour s'y risquer ${ }^{35}$ ». La remarque du prélat est intéressante, car ce n'est qu'après plusieurs refus, des démarches insistantes de Bourget, des pressions de certains "zouaves》 de Montréal que Laflèche décide d'aller à Rome.

En 1874, les prises de position de Laflèche ne lui ont pas encore aliéné la sympathie de ses collègues. Il y a bien eu parfois quelques frottements, avec $\mathbf{M}^{\mathrm{gr}}$ Jean Langevin par exemple. Choqué par le Programme catholique, l'évêque de Rimouski profite de deux incidents mineurs pour admonester Laflèche :

Ainsi V.G. ne se contente pas de me laisser insulter, avec son Archevêque et un autre de ses collègues, par le « Journal des Trois-Rivières », au sortir même de notre réunion épiscopale; Elle vient de plus embaucher un de mes prêtres [Alexis Pelletier], avec lequel j'étais en pourparlers pour lui donner un poste. C'est vraiment une singulière manière d'entendre et de pratiquer la charité, la justice et le respect de l'ordre épiscopal ${ }^{36}$.

32 Cité par Jacques LACouRcière, « Mgr Laflèche et la confédération 》, RSCHEC, 34(1967), p. 66.

33 «Adresse à Notre T.S. Père le Pape Pie IX 》, 13 juillet 1876, Mémoire de l'évêque des Trois-Rivières..., pp. 97-102.

34 Ibid., p. 18.

35 Mgr C. La Rocque à Mgr J. Langevin, 4 déc. 1872, Archives de l'archevêché de Rimouski (AAR), Diocèse de Saint-Hyacinthe, I (1849-1885).

36 Mgr Jean Langevin à Mgr Laflèche, 13 nov. 1871, ASTR, Fonds Lafleche, A 1 L 54. 
Des explications pacifiques de Laflèche permettent heureusement de conjurer l'orage! Taschereau lui-même n'a pas perdu confiance dans l'évêque de Trois-Rivières, et il le consulte souvent; un échange de lettres plus acerbes en 1873-74 annonce cependant les combats homériques des années 1880-85.

Laflèche est déjà, en 1874 , à peu près le même qu'il sera en ces années cruciales. Il a déjà affiché ce que j’appellerais sa théologie politique dans un volume intitulé Quelques considérations sur les rapports de la société civile avec la religion et la famille ${ }^{37}$, publié en 1866 , et dans quelques sermons importants; il n'en déviera guère jusqu'à sa mort. Dans les débats qui secouent la province, il défend avec persistance le point de vue ultramontain, mais il ménage encore celui qui deviendra son bouc émissaire, sa cible de choix, l'archevêque. Est-il moins souple qu'il ne le sera plus tard? Je ne le crois pas, même s'il est en ce moment moins influencé par Luc Désilets, certains chanoines de Montréal ou même l'intransigeant $\mathrm{M}^{\mathrm{gr}}$ Jean Langevin. Dans son diocèse, il est encore adulé par la majorité de la population et du clergé, ses visites pastorales tournent facilement en manifestations populaires avec cavalcades et arcs de triomphe, et sa ville épiscopale pavoise avec enthousiasme à ses retours de voyage, ce qui permet au subtil $\mathrm{M}^{\mathrm{gr}} \mathrm{L}$.-Z. Moreau d'ironiser : "Grandissime fête donc à Trois-Rivières le 11 septembre prochain. Donc adresses sur adresses, car ce paraît être la monnaie courante dont on se sert dans votre cité épiscopale 38 ». Une opposition commence à poindre, particulièrement sur la rive sud: le séminaire de Nicolet a dû mener une dure lutte pour se défendre contre lui à Rome, les blessures ne sont pas cicatrisées et la bataille de la division du diocèse s'annonce déjà; quelques prêtres régimbent contre les taxes ecclésiastiques qui les frappent et ce «jeune» évêque qui ne connaît pas le ministère paroissial; les diocésains "libéraux » ne savourent guère ses diatribes contre eux. Mais tout cela ne fait pas oublier qu'il a sauvé le diocèse de la banqueroute, et il n'a qu'à faire entendre son éloquence prestigieuse pour qu'on boive encore avec suavité ses ordres et ses conseils. Les troubles diocésains sont à venir, comme d'ailleurs les manœuvres moins prudentes du chevalier aveuglé par l'entêtement.

37 Montréal, Eusèbe Sénécal, 1866. 268 p.

38 Cité dans Rolland LITALIEN, Le prêtre québécois à la fin du XIXe siècle, Montréal, Fides, (1970), p. 26. 


\section{4) Les aUtres}

Je ne peux m'empêcher de vous dire au moins quelques mots de $\mathbf{M}^{\mathrm{gr}}$ Jean Langevin.

Nommé titulaire du nouveau diocèse de Rimouski en 1867 , il s'y rend avec armes et bagages, meubles et famille. Il y arrive en bateau, un peu comme Jacques Cartier à Gaspé. Et la famille Langevin s'installe : son frère Edmond comme vicaire général, un autre comme concierge, le beau-frère comme protonotaire; même Hector aurait bien aimé être député de Rimouski, mais il s'est fait battre...

Aux yeux d'Hector, l'influence d'une telle famille ne saurait être que bénéfique: «J'espère que l'arrivée d'un Évêque à Rimouski ne sera [pas] signalée par les Électeurs du Comté par une Élection rouge. Envoyez-nous 1 ou 2 bons bleus, mais pas un seul rouge ${ }^{39}$ ». Dociles, Jean et Edmond vont régulièrement s'intéresser aux élections, au point d'aboutir à la célèbre contestation de Bonaventure en 1876. Aidés par Hector qui fournit le patronage, ils fondent un journal, la Voix du Golfe, qui véhiculera les bonnes idées, et la prose d'Edmond. Ils essayeront, mais en vain cette fois, de faire nommer juge le beau-frère François-Magloire Derome.

Les initiatives de la famille Langevin créent certains remous dans le petit village de Rimouski et une guerre d'usure sévit entre la classe professionnelle et l'évêque. Décrivant à son frère Hector une bataille épique autour du beau-frère Derome, Edmond écrit en 1871 :

Eh ! bien, tu as là un specimen de ce qui se passe tous les jours à Rimouski parmi nos hommes de profession. Et si quelques uns ont une haine invétérée contre leurs supérieurs ecclésiastiques, c'est que leur iniquité a été dénoncé [sic] (en termes généraux) mais très énergiquement du haut de la chaire. Mgr. s'est montré vraiment Évêque ${ }^{40}$.

C'était l'écho d'un commentaire assez semblable fait en 1868 :

Mgr. est dans une assez grande perplexité. Il s'agit pour lui de se déterminer à bâtir, et cependant il trouve tant de froideur et d'indifférence dans la localité qu'il hésite à fixer irrévoca-

39 H. Langevin à Mgr Jean Langevin, 22 juillet 1867, ANQ, Fonds Langevin, B 4, 640.

40 Edmond Langevin à Hector Langevin, 21 janv. 1871, ibid., B 37. 
blement sa résidence. [...] Et avec tout cela pas de zèle dans la population; peu de sympathies dans la gentilhommerie qui est peu religieuse, ${ }^{41}$.

Au sein de l'épiscopat, $\mathrm{M}^{\mathrm{gr}}$ Langevin se voit confier au début les dossiers sur l'éducation, - il avait été principal de l'école normale Laval, - mais il est tellement éloigné qu'on ne peut continuer longtemps cet usage. Il se sent alors isolé, abandonné, et il se plaint souvent de n'être pas tenu au courant des problèmes. En 1871, par amitié pour Québec et par fidélité au parti conservateur, Langevin appuie totalement l'archevêque, mais la contestation de Bonaventure le fera basculer dans le camp ultramontain, où il se révèlera un des plus durs critiques de Taschereau. Avec son clergé, il est alternativement d'une sévérité cassante et d'une bonhommie condescendante, et il laisse finalement le souvenir d'un tyran que le pape doit faire démissionner pour soulager une jeune Église fatiguée. Et pourtant le premier évêque de Rimouski laissait une œuvre importante derrière lui!

L'évêque Charles La Rocque, de Saint-Hyacinthe, mériterait un traitement de faveur, car il est le plus sympathique du groupe épiscopal. En ces jours difficiles, il a essayé d'être un agent pacificateur. Admirateur de $\mathrm{M}^{\mathrm{gr}}$ Bourget, il n'a pas craint de différer d'avis avec lui à propos de liturgie et de prendre parti contre lui en 1871, s'attirant une verte semonce du vieil évêque qui dénonce ce " grand scandale " et proclame que «le monde ne manquera pas de conclure que vous êtes partisan servile de l'autorité séculière; et que, pour servir la cause de quelques amis du monde, qui vous considèrent comme leur appui, vous tournez le dos à vos collègues et biaisez sur les principes que nous sommes chargés de défendre à tout prix 42 ». «Épuisé par les contradictions qui l'avaient accueilli et par le travail qu'il s'était imposé », il meurt en 1875 au moment où son esprit de conciliation aurait été bien utile.

Quant à $\mathrm{M}^{\mathrm{gr}}$ Joseph-Bruno Guigues, d'Ottawa, je me permets d'user envers lui de la même discrétion qu'il a manifestée à propos des problèmes du Québec.

41 Le même au même, 22 nov. 1868, ibid.

42 Cité dans Philippe Sylvan, «La Rocque, Charles》, Dictionnaire biographique du Canada, t. X : de 1871 a 1880, Québec, Les Presses de l'université Laval, (1972), p. 472. 


\section{II. - L'épiscopat dans son ensemble}

J'ai beaucoup insisté sur le caractère et la personnalité de chacun des évêques, parce que j'y vois une bonne explication des conflits qui déchirent l'épiscopat. Mais le tableau, pour être complet, devrait comporter une étude aussi longue sur l'assemblée épiscopale prise en tant que telle. Comme je n'ai pas le temps de le faire, je me contente de quelques notes rapides.

En 1874, l'épiscopat québécois est passablement vieux. Le doyen (Bourget) a 75 ans et le cadet (Langevin) 53 ans. Avec une moyenne de 62 ans, les évêques sont largement au-dessus de l'espérance de vie de leurs contemporains.

Uniformément rassis, ils sortent également d'un même milieu, celui de l'éducation. Quelques-uns (v.g. La Rocque) ont sans doute une solide expérience pastorale, mais tous ont au moins tâté de l'enseignement et la plupart y ont fait carrière. Or, le monde de l'éducation n'est pas précisément le plus libéral de l'époque ou le plus avant-gardiste! Et ses liens avec la réalité sont parfois assez minces... C'est aussi dans des maisons d'enseignement assez semblables que les futurs évêques ont reçu une formation presque identique où les différences tiennent plus de la personnalité de certains professeurs que de doctrines originales. Le système de cooptation par lequel on choisit les evêques accentue encore cette espèce d'uniformité.

Il n'est donc pas surprenant de rencontrer, dans l'épiscopat de 1874 , une unité de pensée qu'on oublie parfois. Sur les questions de fond, tous les évêques québécois pensent de la même façon. Au moment de la discussion de l'infaillibilité pontificale, ils étaient unanimes à appuyer le nouveau dogme; ils dénoncent avec la même vigueur, et le même vocabulaire, les idées modernes, dites révolutionnaires. Il n'est pas inouï que la lettre collective la plus virulente contre le libéralisme ait été écrite par $\mathrm{M}^{\mathrm{gr}}$ Taschereau. Sur l'éducation, à propos des questions sociales, les déclarations épiscopales se ressemblent étrangement à Québec et à Montréal. On ne saurait donc parler d'évêque libéral au Québec et, s'il varie en intensité dans certaines régions, l'ultramontanisme se porte bien partout.

Là où les évêques se distinguent entre eux, c'est dans le domaine de l'agir ou du pratico-pratique. Il y a les idéalistes qui ne veulent pas composer avec la réalité et qui veulent imposer un modèle ("pattern ») sans tenir compte des contingences; comme 
disait Taschereau, « l'idéal de ce qui devrait être tend à [leur] faire oublier la réalité ». Par contre, il y a les réalistes qui, sans renier les principes, croient prudent d'accepter une situation qui n'est pas totalement conforme au modèle idéal; chez eux le sens du relatif prend le pas sur l'intransigeance. Ils pourraient s'approprier cette phrase que le cardinal Barnabo aurait dite à Bourget : «Mgr, je ne suis qu'un simple mortel, je ne plane pas dans les hautes sphères comme Votre Grandeur; vous avez des vues très élevées, moi je suis avant tout un homme pratique $43 \gg$. En un mot, les premiers sont disciples de Pie IX, les autres imitent davantage Léon XIII. Je n'ai pas besoin de vous dire que la plupart des évêques québécois ont appartenu au premier groupe et que seuls peut-être $\mathbf{M}^{\mathrm{gra}}$ Taschereau et $\mathrm{La}$ Rocque peuvent être rangés dans le second.

\section{Conclusion}

$\mathrm{Au}$ terme de cette étude trop courte, je dois vous avouer que plus je fréquente ces évêques du $\mathrm{XIX}^{\mathbf{e}}$ siècle, plus je les trouve intéressants. Derrière la façade de dignité et les conflits extérieurs, on rencontre bientôt des hommes ardents, convaincus, passionnés même, mais humains, très humains (dans tous les sens du terme); ils sont des bourreaux de travail et ils ne comptent jamais leurs peines; leur vie spirituelle est d'une richesse surprenante, même s'ils ne sont pas tous des $\mathbf{M}^{\mathrm{gr}}$ Moreau. Surtout, ils ne sont jamais ennuyeux et, s'ils paraissent parfois ressasser les mêmes rengaines, c'est que nous ne connaissons encore qu'une partie de leur tâche pastorale. Ils ont été de leur temps et ils ont contribué à infléchir la société québécoise dans un sens qui ne nous apparaît pas toujours le meilleur, après coup. Mais les évêques de 1874 n'ont pas besoin de panégyrique, ils ne demandent qu'à être vus, par les historiens, comme ils ont été, dans le milieu où ils ont vécu, avec leurs qualités et leurs misères, leurs vertus et leurs défauts. C'est ce que j'ai essayé de faire bien rapidement, avec mes propres limites et mes préjugés.

Nive Vorsine,

Département d'Histoire, Université Laval.

43 Taschereau à E.-T. Hamel, 1 avril 1865, ASQ, Un. 104-39. 